\section{Single-Tube Method for \\ Determination of F508del \\ Genotype in the CFTR \\ Gene Using Bidirectional \\ PCR Amplification of Specific Alleles}

BioTechniques 34:460-462 (March 2003)

Cystic fibrosis is the most common severe autosomal recessive disorder in Caucasians, with a frequency of 1 in 2000 live births and a carrier frequency of approximately 5\% (1). It is a multi-system disorder that leads to chronic pulmonary and exocrine pancreatic disease, associated with elevated sweat electrolytes and male infertility due to bilateral absence of the vas deferens (2-4). Mutations in the cystic fibrosis transmembrane conductance regulator (CFTR) gene are the genetic cause of this disease (2-4), and their identification allows molecular diagnosis in patients, carrier and prenatal testing in cystic fibrosis families, and population screening (NIH consensus statement, 1997).

The CFTR gene spans $189 \mathrm{~kb}$, with a coding region of $6.2 \mathrm{~kb}$, including 27 exons. More than 1000 mutations have been described in this gene (http://www. genet.sickkids.on.ca/cftr/rptTABLE2. html) (5), with a widespread distribution that complicates direct testing (1). The most common mutation in all populations is a deletion of three nucleotides that results in loss of a phenylalanine in the CFTR protein, at position 508 (F508del). Therefore, strategies for genetic testing in patients/families with cystic fibrosis are often initiated with direct detection of this particular mutation, such as the allele-specific PCR method described by Ballabio et al. (6). This method requires two PCRs for the determination of the genotype at this locus and does not allow the distinction between F508del (69\% of cystic fibrosis alleles in the US) and the less common I507del mutation $(0.31 \%$ in the US) (1), because the primers used for amplification of the mutant allele will match both of these sequences. Individuals with an I507del mutation will erro-

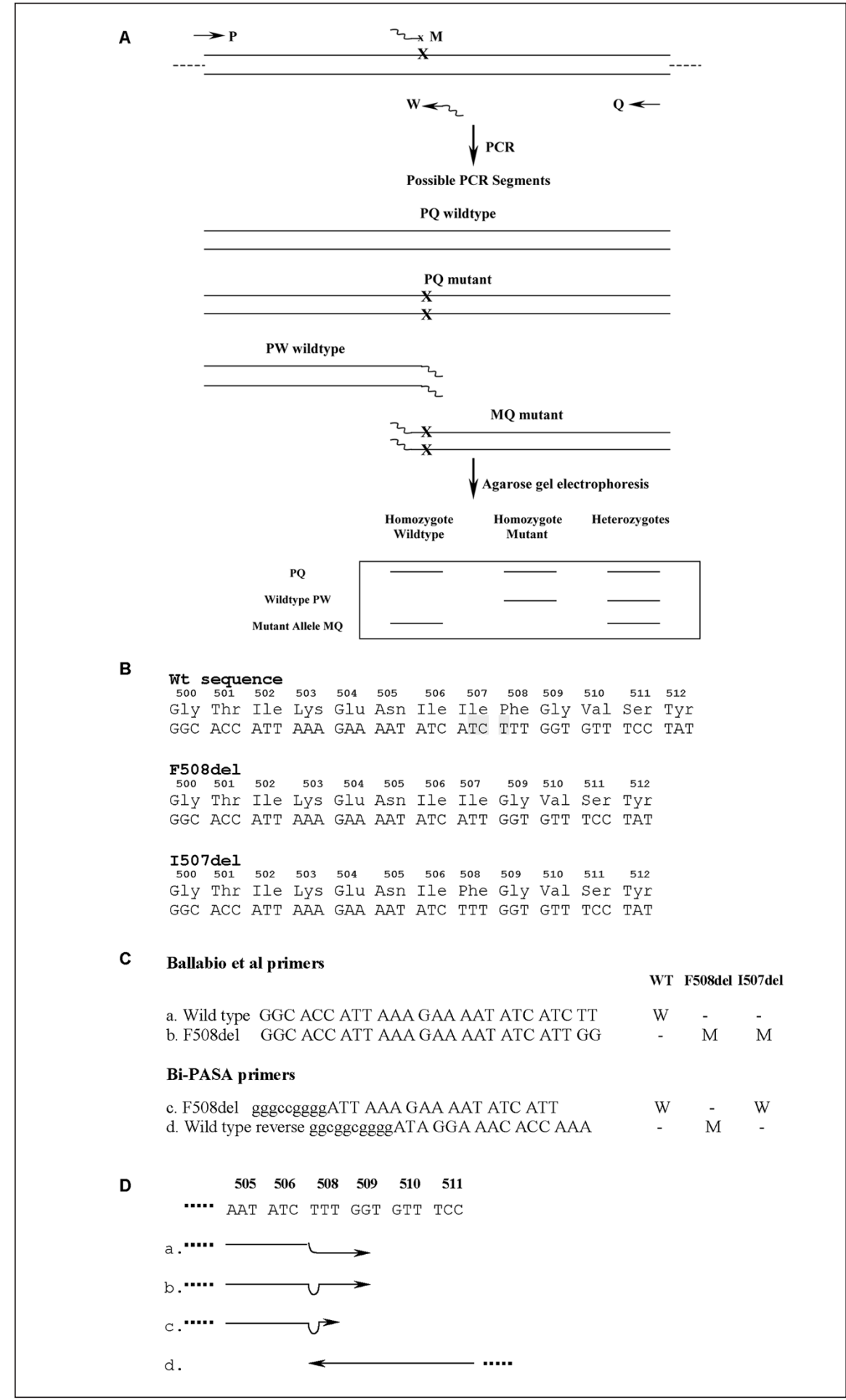

Figure 1. F508del Bi-PASA assay. (A) Principle of the assay. The four primers ( $P, Q$, and $M=F 508$ del mutation; and $\mathrm{W}=$ wild-type) are represented by arrows. $\mathrm{X}$ represents the mutation. Wavy lines on the end of primers represent noncomplementary $5^{\prime}$ tail sequences. The PQ segment serves as a constant reference that confirms the technical success of the assay. (B) Relevant sequences (base numbers according to Reference 3). Three nucleotides deleted at F508del are shaded. (C) Sequence of the Ballabio and the Bi-PASA primers. The Ballabio primers score I507del as the F508del, while only the F508del gives a mutant signal with the Bi-PASA primers. (D) Matching of primers a-d with the I507del sequence. Mismatches are indicated by bends in the line. The Ballabio b primer amplifies the I507del allele because the one mismatch is at -5 from the $3^{\prime}$ end. Primer $\mathrm{c}$ is the Bi-PASA primer specific for F508del that does not allow amplification of I507del because the one nucleotide mismatch is only two nucleotides from the $3^{\prime}$ end. 
Table 1. Primers Used for Bi-PASA of F508dela

\begin{tabular}{|c|c|c|}
\hline Primer & Sequence ${ }^{a}\left(5^{\prime} \rightarrow 3^{\prime}\right)$ & $\mathrm{T}_{\mathrm{m}}\left({ }^{\circ} \mathrm{C}\right)^{\mathrm{b}}$ \\
\hline $\mathrm{P}$ & CTGAATCATGTGCCСTTCTC & 60 \\
\hline Q & CATTCACAGTAGCTTACCCAT & 60 \\
\hline $\mathrm{M}$ & gggccgggggATTAAAGAAAATATCATT & 40 \\
\hline W & ggcggcggggATAGGAAACACCAAA & 40 \\
\hline \multicolumn{3}{|c|}{$\begin{array}{l}\text { aParts of the sequence in lowercase are noncomplementary tails. } \\
\text { b } T_{m} \text { is calculated by the Wallace formula equals } 2^{\circ} \mathrm{C} \text { for each } \mathrm{A} \text { or } \mathrm{T} \text { and } 4^{\circ} \mathrm{C} \text { for } \\
\mathrm{G} \text { or } \mathrm{C} \text {. The } T_{m} \text { is for original genomic DNA. }\end{array}$} \\
\hline
\end{tabular}

neously be typed as F508del with allele-specific PCR (Figure 1). We have developed an improved method for the rapid detection of F508del, using bidirectional PCR amplification of specific alleles (Bi-PASA) (7), which involves an internally controlled, single PCR that $(i)$ distinguishes homozygotes and heterozygotes for F508del and (ii) facilitates more accurate molecular epidemiological analyses because the F508del and the I507del mutations are not confused with each other.

The Bi-PASA method uses a combination of four primers, two outer primers (designated $\mathrm{P}$ and $\mathrm{Q}$ ) and two inner allele-specific primers (designated $\mathrm{M}$ and $\mathrm{W}$ ). In heterozygotes, three segments are amplified: segment M-Q resulting from the mutant allele, segment $\mathrm{P}-\mathrm{W}$ resulting from the wild-type allele, and segment $\mathrm{P}-\mathrm{Q}$, a combined segment of larger size, which serves as an internal control for the reaction (Figure 1). The allele-specific segments are easily distinguishable by agarose gel

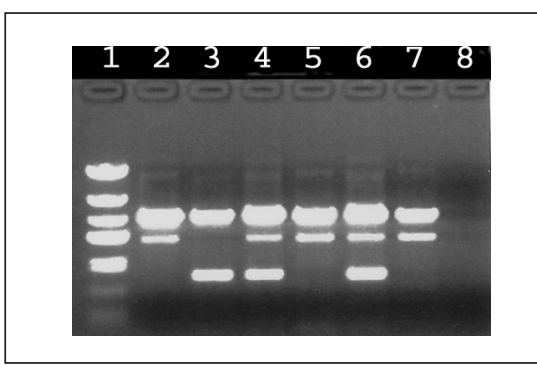

Figure 2. Agarose gel showing results of $\mathrm{Bi}-$ PASA genotyping for F508del. Bi-PASA Assay applied to samples containing wild-type, F508del, and I507del. The assay was performed as described in the text. Lane 1, $\varphi x 174-$ PUC19/HaeIII marker; lane 2, homozygote wildtype; lane 3, homozygote F508del; lane 4, heterozygote F508del; lane 5, heterozygote I507del; lane 6, heterozygote F508del; lane 7, homozygote wild-type; and lane 8, no DNA control. electrophoresis. The two inner primers contain a relatively short allele-specific complementary region and a 10-nucleotide GC-rich 5' tail. The GC-rich tail is used to prevent "megapriming", which occurs when a segment generated by PCR in an earlier cycle acts as a primer for genomic DNA in subsequent cycles $(8,9)$, and to increase the efficiency of self-amplification of the P-W and M-Q segments in later PCR cycles because of an increased region of complementarity (7). In brief, amplification is robust because primer design keeps the different segments from interacting (i.e., PQ is prevented from giving rise to the small segments, $\mathrm{PW}$ or $\mathrm{MQ}$, while PW and MQ are prevented from producing PQ by megapriming) (8).

Primers for Bi-PASA were designed following the guidelines proposed by Liu et al. (7), which accounts for the $T_{m}$ of the complementary regions of the primers and the $T_{m}$ of the PQ segment (Table 1). The Wallace temperature of an oligonucleotide provides an approximate estimate of the $T_{m}$ in $1 \mathrm{M}$ sodium chloride. The current primers are stabilized by magnesium so that the Wallace temperature is only a guideline for the relative stability of two primers under PCR conditions. These primers were designed as per the criteria in Liu et al. (7). The primers were designed with a short complementary sequence and a tail to inefficiently amplify from the genomic DNA template while efficiently amplifying the PCR products that contain the tail sequence. PCR amplification was performed using $1 \mathrm{U}$ TaqGold DNA polymerase (Roche Applied Science, Indianapolis, IN, USA), in a total volume of $25 \mu \mathrm{L} 50 \mathrm{mM} \mathrm{KCl}, 10 \mathrm{mM}$ Tris-HCl, pH 8.3, $2.5 \mathrm{mM} \mathrm{MgCl}_{2}, 200$ $\mu \mathrm{M}$ each $\mathrm{dNTP}$, for 35 cycles of $15 \mathrm{~s}$ at $94^{\circ} \mathrm{C}, 30 \mathrm{~s}$ at $58^{\circ} \mathrm{C}$, and $1 \mathrm{~min}$ at $72^{\circ} \mathrm{C}$, after a 10-min initial period of DNA denaturation and enzyme activation at $94^{\circ} \mathrm{C}$. The inner primers were at a concentration of $0.6 \mu \mathrm{M}$, which is 1.5 -fold more concentrated than the outer primers. The concentrations of the inner primers could vary $0.6-1.2 \mu \mathrm{M}$ and the outer primers, $0.4-0.8 \mu \mathrm{M}$. The annealing temperature of $55^{\circ} \mathrm{C}-60^{\circ} \mathrm{C}$ is compatible with accurate genotyping (data not shown).

The amplified segments have sizes of $587 \mathrm{bp}(\mathrm{P}-\mathrm{Q}), 414 \mathrm{bp}(\mathrm{P}-\mathrm{W})$, and $226 \mathrm{bp}$ (M-Q), and they are readily distinguishable by electrophoresis through a $2 \%$ agarose gel (Figure 2 ).

A blinded analysis was conducted to validate the method using DNA samples from 32 cystic fibrosis patients. All were accurately genotyped as confirmed by sequencing.

In conclusion, F508del genotyping by Bi-PASA is a rapid, internally controlled method that distinguishes homozygotes and heterozygotes and is amenable to large-scale population screening.

We acknowledge helpful discussions from Sebastian Beck and Qiang Liu. We thank John Cohn for help in ascertaining samples for the blinded analysis. Address correspondence to Dr. Steve S. Sommer, Director, Departments of Molecular Genetics and Molecular Diagnosis, City of Hope $\mathrm{Na}$ tional Medical Center, 1500 East Duarte Road, Duarte, CA 91010-3000, USA. email: sommerlab@coh.org

\section{REFERENCES}

1.Welsh, M.J., B.W. Ramsey, F. Accurso, and G.R. Cutting. 2001. Cystic Fibrosis, p. 51215188. In C.R. Scriver, A.L. Beaudet, W.S. Sly, and D. Valle (Eds.), The Metabolic \& Molecular Bases of Inherited Disease. McGraw-Hill, New York.

2.Kerem, B., J.M. Rommens, J.A. Buchanan, D. Markiewicz, T.K. Cox, A. Chakravarti, M. Buchwald, and L.C. Tsui. 1989. Identification of the cystic fibrosis gene: genetic analysis. Science 245:1073-1080.

3.Riordan, J.R., J.M. Rommens, B. Kerem, N. Alon, R. Rozmahel, Z. Grzelczak, J. Zielenski, S. Lok, et al. 1989. Identification of the cystic fibrosis gene: cloning and characterization of complementary DNA. Science 245:1066-1073.

4.Rommens, J.M., M.C. Iannuzzi, B. Kerem, M.L. Drumm, G. Melmer, M. Dean, R. 
Rozmahel, J.L. Cole, et al. 1989. Identification of the cystic fibrosis gene: chromosome walking and jumping. Science 245:1059-1065.

5.Tsui, L.C. 1992. Mutations and sequence variations detected in the cystic fibrosis transmembrane conductance regulator (CFTR) gene: a report from the Cystic Fibrosis Genetic Analysis Consortium. Hum. Mutat. 1:197-203.

6.Ballabio, A., R.A. Gibbs, and C.T. Caskey. 1990. PCR test for cystic fibrosis deletion. Nature 343:220.

7.Liu, Q., E.C. Thorland, J.A. Heit, and S.S. Sommer. 1997. Overlapping PCR for bidirectional PCR amplification of specific alleles: a rapid one-tube method for simultaneously differentiating homozygotes and heterozygotes. Genome Res. 7:389-398.

8.Sarkar, G. and S.S. Sommer. 1990. The "megaprimer" method of site-directed mutagenesis. BioTechniques 8:404-407.

9.Sarkar, G. and S.S. Sommer. 1992. Doublestranded DNA segments can efficiently prime the amplification of human genomic DNA. Nucleic Acids Res. 20:4937-4938.

10.Kerem, B.S., J. Zielenski, D. Markiewicz, D. Bozon, E. Gazit, J. Yahav, D. Kennedy, J.R. Riordan, et al. 1990. Identification of mutations in regions corresponding to the two putative nucleotide (ATP)-binding folds of the cystic fibrosis gene. Proc. Natl. Acad. Sci. USA $87: 8447-8451$

Received 15 October 2002; accepted 19 December 2002.

P. Maciel ${ }^{1,2}$, J. Yan ${ }^{1}$, J. Feng ${ }^{1}$, F. Accurso ${ }^{3}$, and S. Sommer ${ }^{1}$ ${ }^{1}$ City of Hope National Medical Center/Beckman Research Institute

Duarte, CA, USA

${ }^{2}$ University of Porto

Porto, Portugal

${ }^{3}$ Children's Hospital

Denver, CO, USA

\section{High-Throughput Inducible Expression of Transgenes at the Hprt Gene in Mouse Embryonic Stem Cells}

BioTechniques 34:462-468 (March 2003)

Overexpression and/or ectopic expression of gene products has led to important discoveries about gene product function; unfortunately, the expression of transgenes has been difficult and unreliable because of our poor understanding of transcription. Pronuclear injection has been the most common method to introduce transgenes into mouse embryos; however, the problems that arise from pronuclear injection are well described (1). First, chromosomal deletions, inversions, and duplications are frequently generated with transgene integration. These alterations could potentially mutate nearby genes, resulting in an unwanted phenotype that complicates analysis of the transgene product (2). Second, variable levels and patterns of transgene expression are observed between founder mice due to concatemerization of the transgene and chromatin position effects (3). Third, mosaic expression of the transgene may occur because of position-effect variegation $(4,5)$, methylation (6), or intrachromosomal recombination within the transgene concatemer (7). Transgenic mice derived from multiple founders must be analyzed to control for these variables, substantially increasing the resources devoted to an experiment. Transgenes can also be introduced directly into cells or mouse embryos by a variety of techniques including retroviral and adenoviralmediated gene transfer and DNA:lipopolyamine-mediated gene transfer. However, these procedures still present many of the same problems as pronuclear injection, in particular, sustained expression of the transgene.

To address these problems, a procedure has been developed termed targeted transgenesis (8). This procedure introduces a single copy of a transgene into a known location and relies on manipulation of embryonic stem (ES) cells in tis- sue culture. Different methods to achieve targeted transgenesis have been developed that include targeting a transgene to the hypoxanthine phosphoribosyltransferase (Hprt) gene (9) and to other loci, as reviewed by Jasin et al. (8). Hprt was chosen because it is a housekeeping gene, expressed in all cells, and functions in the purine salvage pathway. Thus, chromatin effects are likely minimal. In addition, Hprt is on the $\mathrm{X}$ chromosome and therefore is hemizygous in male cells. Disruption of Hprt can be selected in 6-thioguanine (TG) media and expression of Hprt can be selected in hypoxanthine-aminopterin-thymidine (HAT) media. This selection strategy enables high-throughput transgenesis by gene targeting. The fact that all transgenes are positioned at the exact same location in the genome controls for unknown variables of position effect that accompany random integration.

Previously, we have targeted transgenes to Hprt in mouse ES cells; this approach mutated the Hprt gene (9). Our data showed that a reporter was expressed in greater than $85 \%$ of Hprtmutant ES cell clones. In addition, another reporter exhibited the correct temporal and tissue-specific expression pattern of the promoter in mouse embryos. Thus, targeted transgenesis was successful at the Hprt gene.

Here our goal is to establish a highthroughput method for inducible transgene expression in cells and mice with the tetracycline responsive system (10). The tet-responsive activator system is composed of the tet repressor fused to the activation domain of virion protein 16 (VP16) of herpes simplex virus. This transactivator, called $t \mathrm{TA}$, stimulates transcription of a minimal promoter fused to tet operator sequences $($ tet $\mathrm{O})$. The minimal promoter is the minimal immediate early promoter of the cytomegalovirus (mCMV) juxtaposed to the tet $O$ sequences (tet $O$ mCMV). Inducible expression is achieved by the use of a derivative of tetracycline called doxycycline, which binds and inactivates $t \mathrm{TA}$. Thus, $t \mathrm{TA}$ activates transcription, and doxycycline prohibits this activation.

A luciferase reporter (11), under the regulation of the tet $O$-CMV promoter, was tested for inducible expression when targeted to the Hprt gene. We 